\title{
ARQUEOLOGÍA ANDALUSÍ EN BALAGUER (LÉRIDA)
}

Por

JOSEP GIRALT BALAGUERÓ

Con la creación del Servei d'Arqueologia dentro de la Direcció General del Patrimoni Cultural el 4 de diciembre de 1980 (D. O. G núm. 105), la arqueología en Catalunya entró en una e tapa decisiva que durante los últimos años ha visto cómo se recogian los frutos de dicho trabajo. También ha sido un momento muy importante para la arqueologia andalusi en la zona más septentrional del Sharq al-Andalus, y concretamente Balaguer ha gozado de una posición privilegiada. Hay que decir, sin embargo, que hasta este último año no se han emprendido excavaciones en otros yacimientos, como ha sido el caso de Tortosa. De todos modos, el factor que quizás haya frenado. o mejor dicho, el que no ha potenciado la arqueología islámica ha sido el interés marcado por conocer las raices cristianas de Catalunya, dejando de lado el sustrato musulmán que poseen nuestras tierras.

\section{Antecedentes de la arqueología andalusi en Balaguer}

El único yacimiento en el cual se ha trabajado anteriormente es el conocido Castell Formós. En el año 1967, al efectuar unas obras de consolidación del sector oriental de la muralla del castillo, aparecieron una serie de restos arquitectónicos y cerámicos entre los que destacaban las yeserias islámicas del siglo $X \mathrm{X}$. Éstas son las que estudió el arquitecto $\mathrm{C}$. Ewert, del Instituto Arqueológico Alemán de Madrid, que fue invitado para hacerlo el año 1968 (1).

A partir de este momento, y en vista de los resultados que se podian sacar, la Dirección General de Bellas Artes envió el año 1970 al eminente epigrafista don Manuel Ocaña para que hiciera unas prospecciones al yacimiento. De dichos trabajos nunca se ha publicado nada. Al año siguiente fue el señor Luis Díez-Coronel, que ya habia participado y controlado los trabajos del año 1967. a la vez que los había dado a conocer en distintos trabajos (2), quien excavó el sector norte de la fortificación, sin que tampoco se conozcan los resultados. La última excavación de la primera época del Castell Formós fue dirigida por el señor Juan Zozaya, excavando en el extremo suroriental, trabajos que tampoco han visto su publicación (3).

(1) EWERT, C., "Hallazgos islámicos en Balaguer y la Aljafería de Zaragoza", E. A, E. 97, Madrid, 1979

(2) DIEZ CORONEL, L., "Fortificaciones árabes en Balaguer (Léridal con un palacio del siglo Xl», XII $C$. $A$. $N$. lóem "La a ácazaba de Balaguer $y$ su palacio árabe del siglo XI". Irerda, XXIX, 1969 . Idem, "El castell dels Comtes d'Urgell, alcaçaba ătab», Herda, XXXIV, 1973.

(3) Agradecemos ta información dada por el señor Juan Zozaya, así como su continuo apoyo $v$ consejos 
Hasta el año 1982 todo quedó paralizado y con los traspasos de competencias a la Generalitat de Catalunya, el Servei d'Arqueologia, juntamente con el Museo Municipal (ahora Museu Comarcal de la Noguera), decidieron emprender de nuevo los trabajos arqueológicos en el Castell Formós, así como también trazar un plan conjunto que afectara a otros puntos del conjunto andalusí que es la ciudad de Balaguer: Pla d'Almatà y cásco urbano.

\section{Castell Formós}

En este yacimiento se han realizado ya tres campañas, las dos primeras en el interior, mientras la tercera ha tenido como marco la zona exterior norte de la fortificación.

La única sala del castillo que se conserva intacta es una cámara subterránea, de planta rectangular y cubierta de medio punto, con óculo central en la bóveda, y a la que se accede por unas escaleras. Probablemente en un momento inicial fuera un aljibe, aunque posteriormente, en el siglo XVII, se convirtió en lo que actualmente es. Partiendo de ella y teniendo como referencia las catas abiertas por el señor Zozaya, se hizo un cuadro inicial de $19 \times 12$ metros (N/S-E/O), posteriormente ampliado por su cara oeste entre 3 y 7 metros. Nuestra intención era encontrar las estructuras que estarian relacionadas $y$ adosadas a la sala, y de este modo partir de un conjunto rico de elementos del palacio. Eso no fue asi, ya que las estructuras aparecían cortadas hasta los cinco metros en dirección oeste y a lo largo de to do el eje norte-sur de la cata. Esto nos hizo pensar en una posible reparación de toda la sala subterránea, hecho que hubiera comportado la destrucción de todos los elementos existentes en esta zona; esta obra se produciría en una fecha posterior al 1627 , ya que la aparición de un Ardit con esta fecha en los niveles de relleno de la cámara, nos daría la fecha más antigua para dicha reparación.

En el extremo norte del cuadro se localizó parte de una habitación importante precedida de un corredor con pavimento de yeso y con acceso a ella por dos escalones hechos de sillares islámicos reaprovechados. Decimos que es una sala relevante porque la cubierta de la zona excavada, que por cierto estaba en muy mal estado de conservación, presentaba restos de pintura en el envigado, con una alternancia de cuadrados negros y rojos. Además, el nivel de destrucción de dicha sala era muy rico en restos de decoración arquitectónicos a base de alicatados de finales del XIV.

Pero sin ningún tipo de dudas, la estructura más interesante y espectacular apareció en el sector oeste. Se trata de una gran alberca de planta rectangular alargada, construida de ladrillos con alternancia cada dos metros de estribos de piedra para dar más consistencia a la obra. No sabemos aún cuáles serán sus dimensiones totales, ya que falta por excavar su extremo meridional, pero actualmente tiene $14 \times 3,70 \times 1,50$ metros. El pavimento es de piedra arenisca de buena calidad. Desgraciadamente la cara occidental está casi totalmente saqueada, aunque nos han quedado los elementos necesarios para hacer su reconstrucción. Al exterior, dos conducciones de piedra llevarían el agua hacia el probable jardín que habría en este sector del palacio. El trazado inicial de la alberca hay que situarlo en el siglo XI como elemento del palacio taifa, y por tanto contemporánea de las yeserías.

Esta campaña demostró que todo el complejo palaciego está perfectamen- 
te adaptado al terreno, y que se desarrolla a base del aterrazamiento de las distintas salas o elementos. De esta manera, la parte central del tosal estaría ocupada por las cámaras más altas (de aquí el hecho comprobado en nuestras excavaciones de que estas zonas han recibido un fuerte saqueo moderno), mientras que en las vertientes se irían escalonando. La comunicación de todo el complejo se realizaría por medio de escaleras o rampas. El ejemplo más claro es la caja de escaleras que comunicaba la habitación señorial norte con el posible jardín de la alberca que está a unos dos metros por debajo de su nivel.

\section{ZONA EXTERIOR NORTE. CAMPAÑA 1984}

Formando parte del Programa de Recuperació de Jaciments Arqueológics del Pla de Solidaritat amb I'Atur se ha realizado una actuación en la cara exterior norte del castillo. La idea inicial tenía como provecto una limpieza general de toda la muralla y torres. Con posterioridad, y en vista de que el conjunto tenía unas posibilidades arqueológicas muy interesantes, se decidió realizar una excavación a lo largo de todo el eje este-oeste de la fachada norte. Hay que decir que esta campaña ha sido la más espectacular e interesante para el conocimiento de la fortificación de época musulmana, a la vez que ha aportado toda una serie de datos que esclarecerán un poco más la tan discutida polémica en torno a las construcciones emirales. Por un documento árabe, sabemos que Lubb b. Muhammad, después de una campaña contra el condado de Barcelona en la que derrota y da muerte a Guifré el Pilós, se retira a Balaguer y comienza a construir el castillo; esto sucedía en el $897 / 898$ (4). Esta fecha concordaba muy bien con to que hasta ahora se conocía de la fachada norte fver estudio de $C$. Ewert), y más aún si lo relacionamos con otras construcciones fechables en la misma época. A partir de ahora tendremos que dar la misma cronología emiral para los dos elementos nuevos aparecidos: dos torres albarranas y gran foso excavado en la terraza cuaternaria.

\section{Plà d'Almatà}

La importancia militar de Balaguer en la frontera superior de al-Andalus se ve reforzada por un elemento que ya era conocido superficialmente (5), pero que después de los últimos trabajos a rqueológicos realizados se ha podido confirmar definitivamente. Se trata del Plà d'Almatà, extensa llanura situada al noroeste del castillo con una superficie aproximada de veinte hectáreas. Tanto la cara norte como la oeste están fuertemente amuralladas con la presencia de tores de planta cuadrada que se van alternando con tramos de muralla. La técnica constructiva es mixta y va combinando los sillares, el tapial y la mampostería.

Como ya demostró la campaña del 1983, este yacimiento presenta problemas a la hora de buscar cuál era su función, debido sobre todo a que aún no están estudiados los emplazamientos andalusis de las zonas fronterizas y mucho menos los asentamientos de tipo militar en la Marca Superior. El Plà d'Almatà presenta la disyuntiva de ser la primitiva ciudad o bien un campamento militar estable debido a su situación estratégica.

(4) ANTUÑa, M. M., Chronique de Règne du Calife Umaryade 'Abd Alläh à Cordoue, Paris, 1937, p. 126

(5) Ver notas 1 ₹ 2 
La excavación dio como resultado la aparición de una serie de estancias rectangulares, construidas con un zócalo de guijarros de río de una altura aproximada entre 30 y 50 centímetros, con paredes de tapial y cubierta de tejas. Parece que los marcos de las puertas y las paredes interiores irían rebozados con un mortero de yeso y cal. Desgraciadamente sobre el urbanismo aún es pronto para avanzar conclusiones al respecto.

La vida de este yacimiento es muy larga, y mientras no se pueda concretar un posible asentamiento anterior, hay que decir que va desde la invasión musulmana (siglos VIII-IX) hasta la conquista cristiana de Balaguer (finales del $\mathrm{XI}$ hasta 11051. Las cronologías más altas vienen dadas por dos elementos diferentes. El primero sería de tipo constructivo, mientras el segundo de tipo cerámico. La zona occidental de la muralla que encierra el llano presenta una construcción mixta de la que, a nosotros, nos interesan los tramos levantados con sillares. Éstos están colocados, todos, a tizón, son un poco almohadillados, están dispuestos a hueso con juntas muy finas, y tienen unas dimensiones aproximadas de 50 centímetros de altura por $110 / 120$ de grueso. En algunos lugares presentan encajes irregulares con la finalidad de dar más consistencia al muro. Es muy clara la diferencia constructiva entre esta muralla y la del castillo, éste muy bien datado en los años posteriores al 897/98; por tanto, parece que la fortificación del Plà d'Almatà es más antigua y de esta forma nos podríamos atrever a dar la cronología general antes citada teniendo en cuenta que es una datación relativa y que una excavación de la muralla nos dará datos mucho más concretos.

El segundo elemento de cronología alta la dan los materiales recuperados en silos (por ahora nunca en niveles de ocupación). Se trata de cerámicas grises con pastas y técnicas casi idénticas a las aparecidas en el poblado visigodo de Bobalá, del que sabemos muy bien el momento de destrucción: principios del siglo VIII, siempre antes del 720 (6). Estas cerámicas, además, están relacionadas con otras decoradas a base de motivos impresos bajo cubierta melada que también podrian tener estas cronologias de siglos VIII-IX.

El último momento de ocupación es el final del siglo XI, y da la impresión de que no hubo destrucción de la zona habitada, sino más bien un rápido abandono ocasionado posiblemente por la presión que las tropas cristianas realizaban sobre Balaguer, ya a principios del XII. De esta manera la excavación es muy rica en cuanto a material cerámico esparcido por toda la superficie de las casas. Prácticamente están presentes todas las técnicas cerámicas conocidas para esta época, desde las piezas de lujo hasta las de uso común, que por cierto son las más numerosas. Creemos que este yacimiento será uno de los puntos básicos para conocer muy bien el mundo de la cerámica andalusí de nuestras tierras.

Concluyendo, podemos decir que estamos ante de un yacimiento que nos podrá dar una visión clara y continua de los cuatro siglos en que Balaguer estuvo bajo el dominio de los musulmanes.

\section{Casco urbano}

La arqueología andalusí de Balaguer ha visto crecer su riqueza documental

(6) TUSET, F., L'arqueologia a Cata/unya, avui. Barcelona, 1982, p. 154 
con la realización de las primeras excavaciones urbanas que han aportado nuevas perspectivas de cara al futuro. Éstas parecen confirmar la hipótesis de que la Balaguer islámica nace, como núcleo urbano, en el siglo $X$.

A raiz de la construcción del colector general de la calle del Pont, aparecieron los restos del primer muro de contención del río que uniría el puente con la puerta de San Salvador, de la que no queda absolutamente nada, pero que, después de esto, hay que pensar en una primera construcción andalusí. El tramo mejor conservado tiene una longitud aproximada de treinta metros y en él se pueden apreciar claramente tres momentos de construcción. El primero es el más interesante para nosotros, pues corresponde a la construcción de época musulmana. Conocemos cuatro hiladas formadas por sillares rectangulares de muy buena factura, dispuestos generalmente a soga con algunas alternativas a tizón, colocados casi a hueso con una fina junta a base de mortero de cal. Por problemas que no vienen al caso, no se pudo averiguar nada de su cimentación, así como tampoco ningún corte transversal, fac tores ambos que hubieran servido para definir mucho mejor esta construcción.

El hecho de que a mediados del siglo $X$ se tenga la intenciór de canalizar e! río nos puede hacer pensar en que se intenta evitar las inundaciones de agua, elemento indicativo que podría ser consecuencia del inicio del casco urbano Por otro lado, existe un elemento que corrobora esta afirmación; mos referimos a la acequia del Cup, que corre paralela al muro de contención del río por su cara oeste y que también es de construcción musulmana, al menos el primer tramo subterráneo, construido totalmente en piedra. Aunque ahora está en proceso de estudio sabemos que ya existía en el 1094 y tenia cerca la mezquita de Avimoni, a la vez que todo el núcleo urbano musulmán se levantaba en esta zona (7).

Esperemos que los próximos trabajos arqueológicos que se van a realizar en este sector del casco antiguo, aporten más luz sobre la Balaqi de los siglos $X$ $y X I$.

(7) SANAHUJA, F. Pere, Hisrória de la ciutat de Bataguer, Balaguer, 1984, p 182 


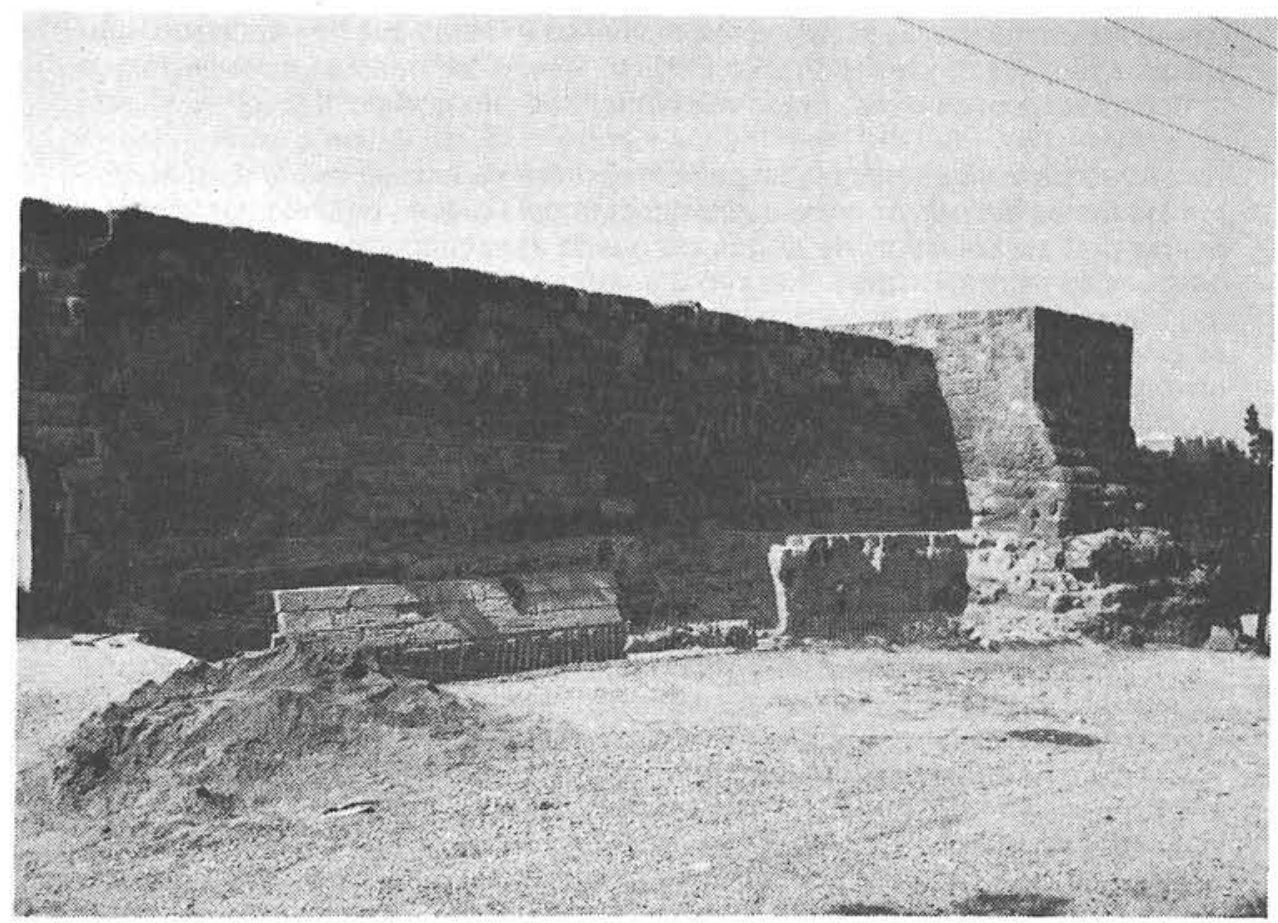

1. - Fachada norte del Castell Formós.

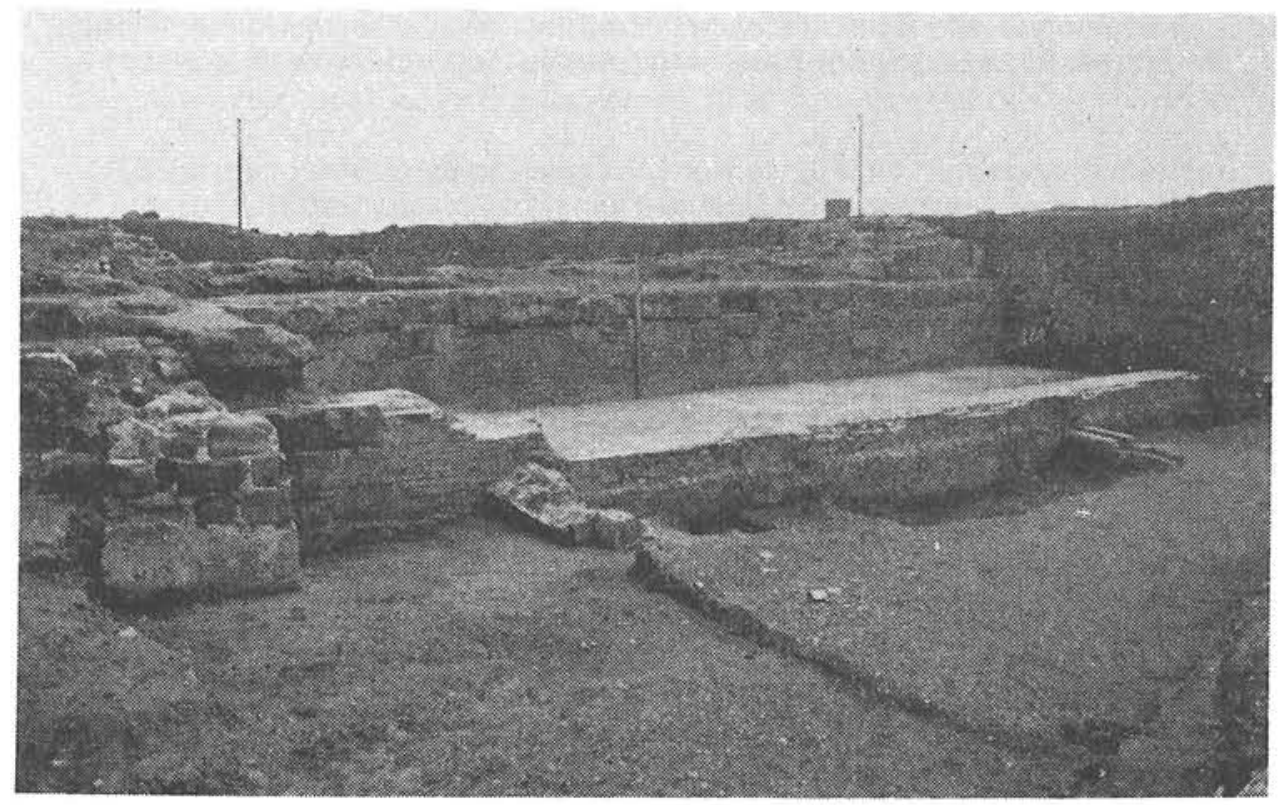

2. - Vista general de la campaña de excavaciones de 1982 en el Castell Formós con la alberca en primer plano. 
ARQUEOLOGIA ANDALUSÍ EN BALAGUER... / Giralt

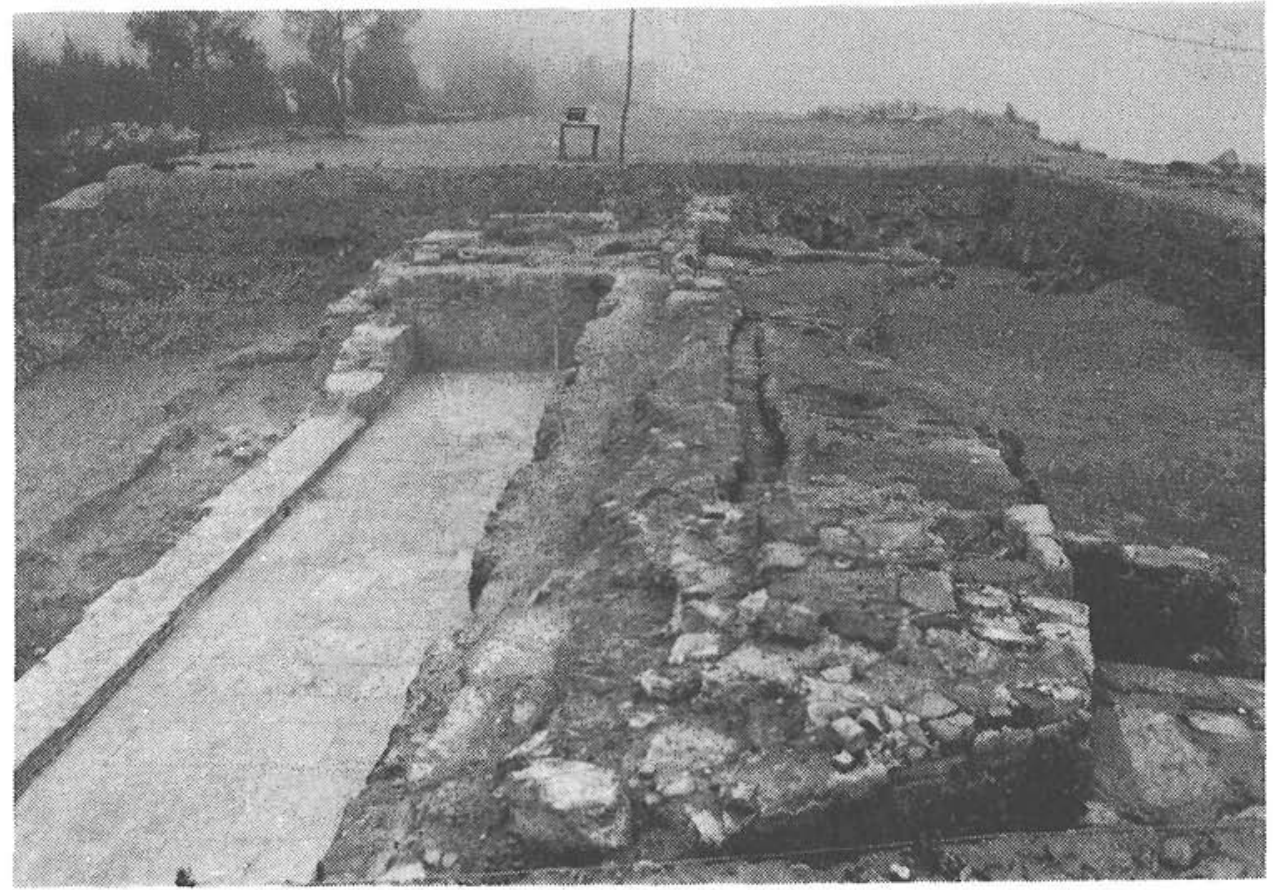

3.-Otra vista de la campaña del 1982

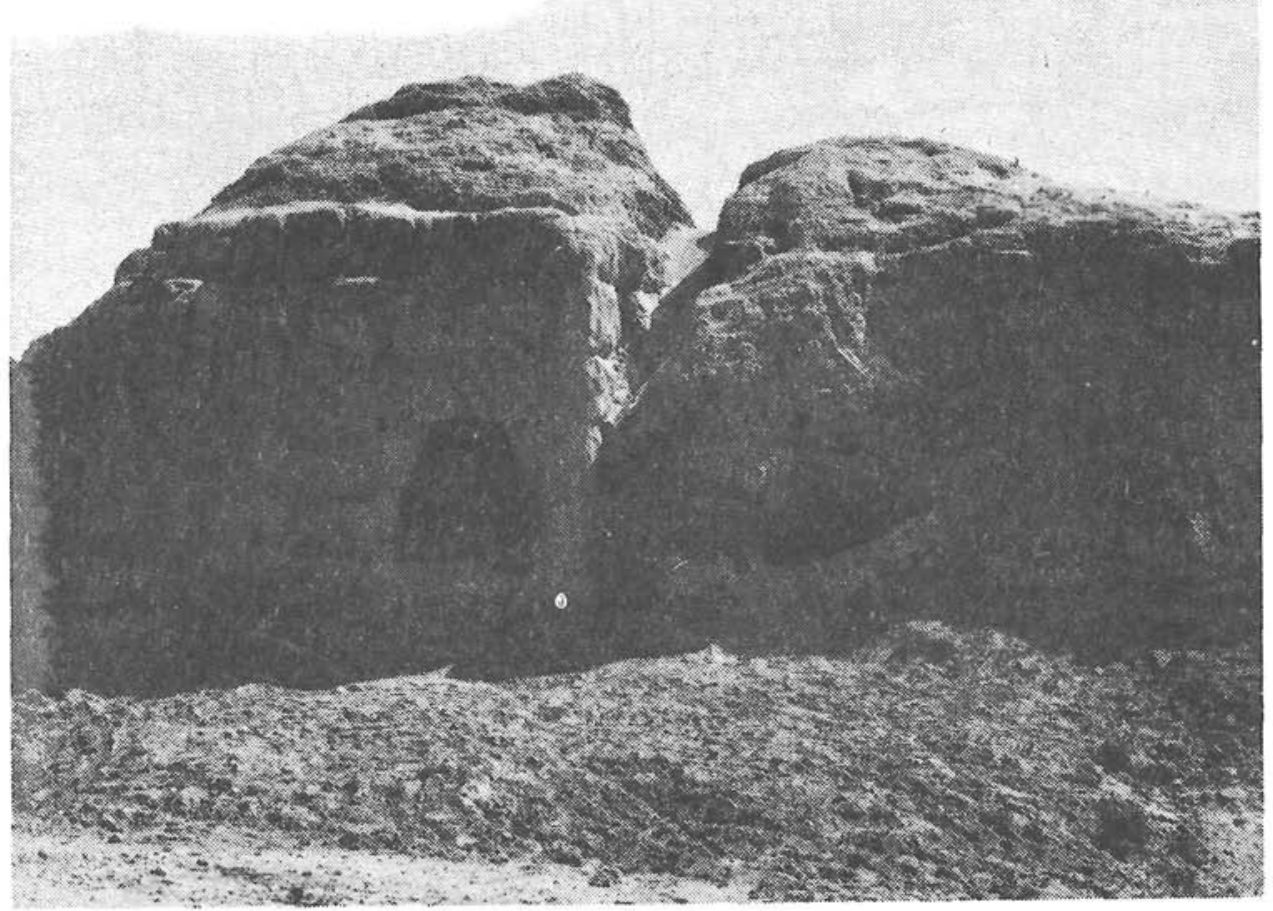

4. - Pla d'Almatà. Una de las torres occidentales del recinto fortificado. 


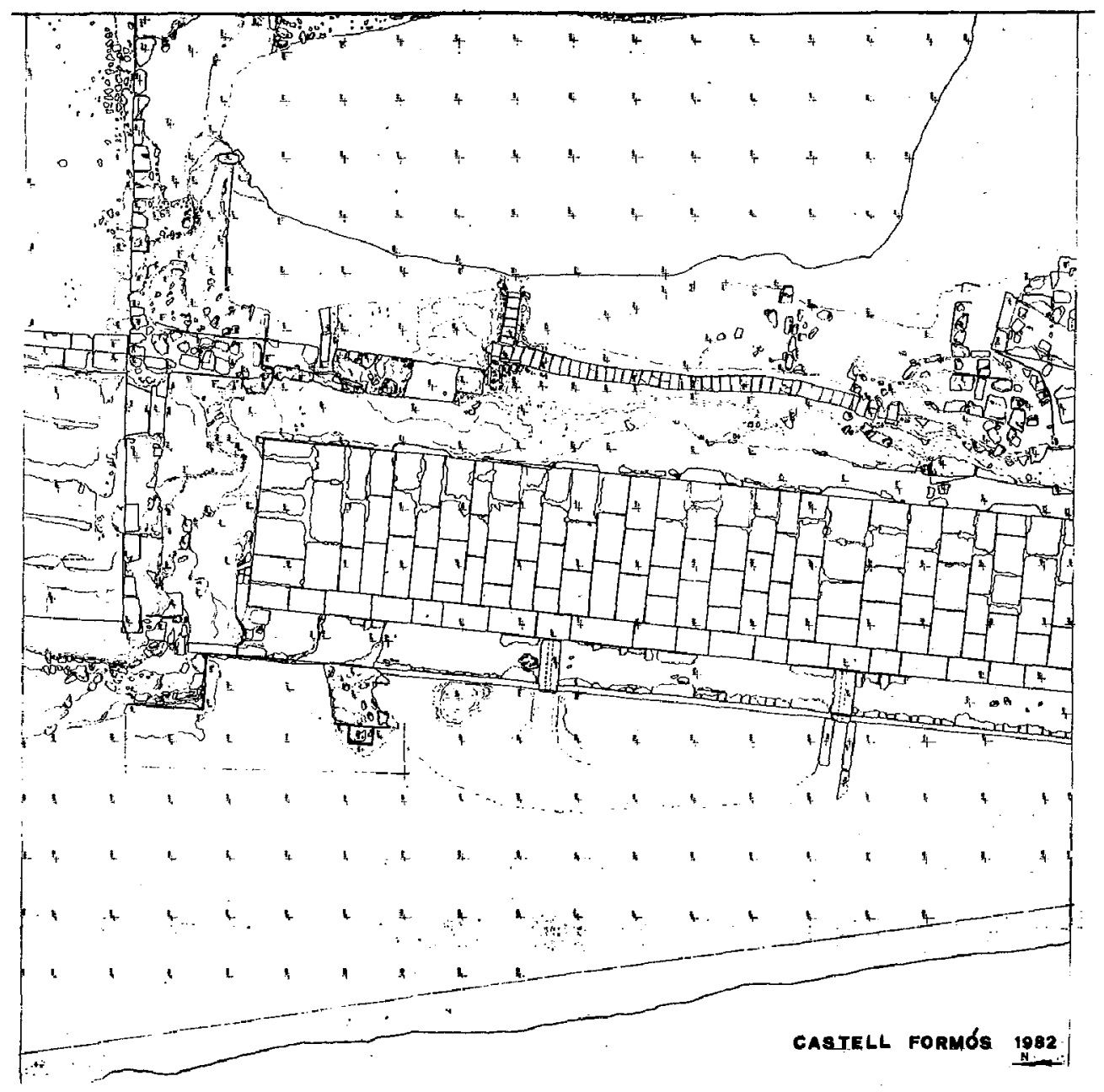



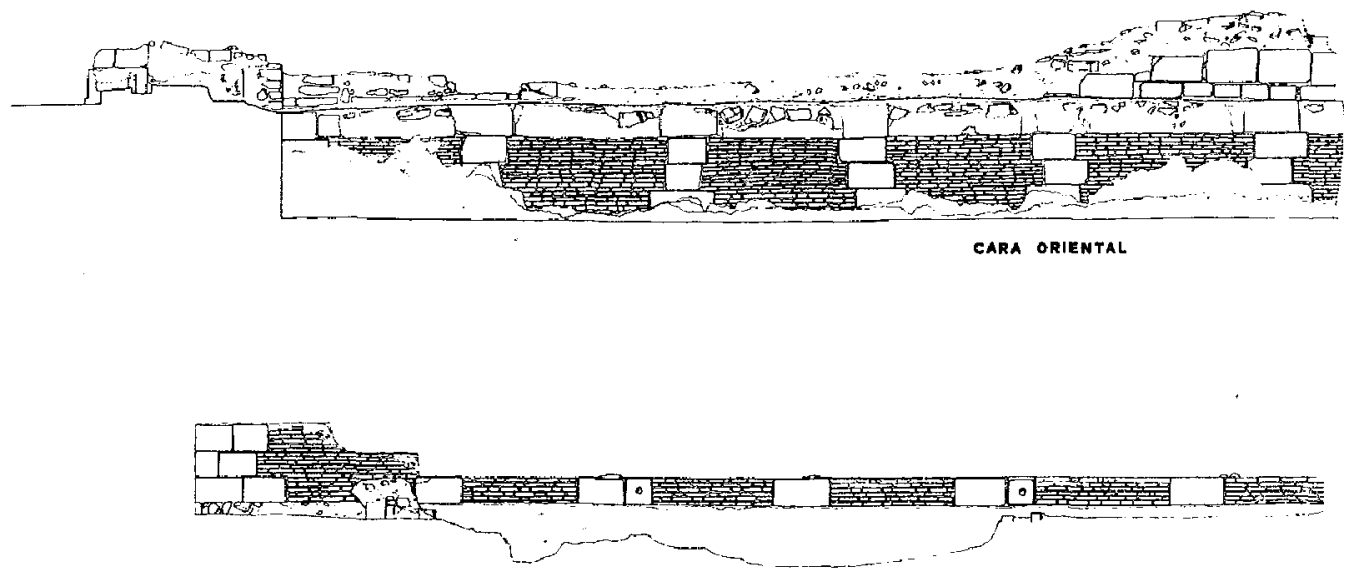

CARA OCCIDENTAL

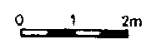

